\title{
Stochastic Dynamics of an SIRS Epidemic Model with Ratio-Dependent Incidence Rate
}

\author{
Yongli Cai, ${ }^{1,2}$ Xixi Wang, ${ }^{1}$ Weiming Wang, ${ }^{1}$ and Min Zhao ${ }^{3}$ \\ ${ }^{1}$ College of Mathematics and Information Science, Wenzhou University, Wenzhou 325035, China \\ ${ }^{2}$ School of Mathematics and Computational Science, Sun Yat-Sen University, Guangzhou 510275, China \\ ${ }^{3}$ College of Life and Environmental Science, Wenzhou University, Wenzhou 325035, China
}

Correspondence should be addressed to Weiming Wang; weimingwang2003@163.com

Received 21 April 2013; Revised 23 May 2013; Accepted 23 May 2013

Academic Editor: Mark A. McKibben

Copyright (c) 2013 Yongli Cai et al. This is an open access article distributed under the Creative Commons Attribution License, which permits unrestricted use, distribution, and reproduction in any medium, provided the original work is properly cited.

\begin{abstract}
We investigate the complex dynamics of an epidemic model with nonlinear incidence rate of saturated mass action which depends on the ratio of the number of infectious individuals to that of susceptible individuals. We first deal with the boundedness, dissipation, persistence, and the stability of the disease-free and endemic points of the deterministic model. And then we prove the existence and uniqueness of the global positive solutions, stochastic boundedness, and permanence for the stochastic epidemic model. Furthermore, we perform some numerical examples to validate the analytical findings. Needless to say, both deterministic and stochastic epidemic models have their important roles.
\end{abstract}

\section{Introduction}

Since the pioneer work of Kermack and McKendrick [1], mathematical models are used extensively in analyzing the spread, and control of infectious diseases qualitatively and quantitatively. The research results are helpful for predicting the developing tendencies of the infectious disease, for determining the key factors of the disease spreading, and for seeking the optimum strategies for preventing and controlling the spread of infectious diseases [2]. And in modeling communicable diseases, the incidence function has been considered to play a key role in ensuring that the models indeed give reasonable qualitative description of the transmission dynamics of the diseases [3-7].

Let $S(t)$ be the number of susceptible individuals, $I(t)$ the number of infective individuals, and $R(t)$ the number of removed individuals at time $t$, respectively. We consider the general SIRS epidemic model:

$$
\begin{aligned}
& \frac{d S}{d t}=b-d S-H(I, S)+\gamma R, \\
& \frac{d I}{d t}=H(I, S)-(d+\mu+\delta) I,
\end{aligned}
$$

$$
\frac{d R}{d t}=\mu I-(d+\gamma) R
$$

where $b$ is the recruitment rate of the population, $d$ is the natural death rate of the population, $\mu$ is the natural recovery rate of the infective individuals, $\gamma$ is the rate at which recovered individuals lose immunity and return to the susceptible class, and $\delta$ is the disease-induced death rate. And the transmission of the infection is governed by an incidence rate $H(I, S)$.

In [8], Liu et al. proposed the general saturated nonlinear incidence rate:

$$
H(I, S)=S g(I), \quad g(I)=\frac{k I^{l}}{1+\alpha I^{h}},
$$

where the parameters $l$ and $h$ are positive constants, $k$ the proportionality constant, and $\alpha$ is a nonnegative constant, which measures the psychological or inhibitory effect. $k I^{l}$ measures the infection force of the disease, and $1 /\left(1+\alpha I^{h}\right)$ measures the inhibition effect from the behavioral change of the susceptible individuals when their number increases or from the crowding effect of the infective individuals. And the other nonlinear incidence rates are considered in [6, 9-19]. 
Note that the infectious force $g(I)$ of classical disease transmission models typically is only a function of infective individuals. But in the transmission of communicable diseases, it involves both infective individuals and susceptible individuals. Thus, Yuan et al. [18, 19] studied the infections force function with a ratio-dependent nonlinear incident rate which takes the following form:

$$
g\left(\frac{I}{S}\right)=\frac{k(I / S)^{l}}{1+\alpha(I / S)^{h}}
$$

And in [19], Li et al. focus on an epidemic disease of SIRS type, in which they assume that the infectious force takes the form of (3) with $l=1$ and $h=1$, and the model is as follows:

$$
\begin{gathered}
\frac{d S}{d t}=b-d S-\frac{k I S}{S+\alpha I}+\gamma R, \\
\frac{d I}{d t}=\frac{k I S}{S+\alpha I}-(d+\mu) I, \\
\frac{d R}{d t}=\mu I-(d+\gamma) R,
\end{gathered}
$$

where all the parameters are nonnegative and have the same definitions as in model (1).

From the standpoint of epidemiology, we are only interested in the dynamics of model (4) in the closed first quadrant $\mathbb{R}_{+}^{3}=\{(S, I, R): S \geq 0, I \geq 0, R \geq 0\}$. Thus, we consider only the epidemiological meaningful initial conditions $S(0)>0$, $I(0)>0, R(0)>0$. Straightforward computation shows that model (4) is continuous and Lipschizian in $\mathbb{R}_{+}^{3}$ if we redefine that when $(S, I, R)=(0,0,0), d S / d t=b, d I / d t=0$, $d R / d t=0$. Hence, the solution of model (4) with positive initial conditions exists and is unique.

It is clear that the limit set of model (4) is on the plane $S+I+R=b / d$, and the model can be reduced to the following:

$$
\begin{gathered}
\frac{d S}{d t}=\left(b+\frac{\gamma b}{d}\right)-(d+\gamma) S-\gamma I-\frac{k I S}{S+\alpha I}, \\
\frac{d I}{d t}=\frac{k I S}{S+\alpha I}-(d+\mu) I,
\end{gathered}
$$

when $(S, I)=(0,0), d S / d t=b+(\gamma b / d), d I / d t=0$.

For mathematical simplicity, let us nondimensionalize model (5) as in [19] with the following scaling:

$$
x=\frac{d(d+\mu)}{b(d+\gamma)} S, \quad y=\frac{d \gamma}{b(d+\gamma)} I, \quad \tau=(d+\mu) t .
$$

We still use variable $t$ instead of $\tau$, and model (5) takes the following form:

$$
\begin{gathered}
\frac{d x}{d t}=1-q x-y-\frac{a x y}{x+p y}, \\
\frac{d y}{d t}=\left(\frac{R_{0} x}{x+p y}-1\right) y,
\end{gathered}
$$

where $q=(d+\gamma) /(d+\mu), p=\alpha(d+\mu) / \gamma, a=k / \gamma$ are positive constants. $R_{0}=k /(d+\mu)$ is the basic reproduction number. And when $(S, I)=(0,0), d x / d t=1, d y / d t=0$.

On the other hand, if the environment is randomly varying, the population is subject to a continuous spectrum of disturbances [20,21]. That is to say, population systems are often subject to environmental noise; that is, due to environmental fluctuations, parameters involved in epidemic models are not absolute constants, and they may fluctuate around some average values. Based on these factors, more and more people began to be concerned about stochastic epidemic models describing the randomness and stochasticity [22-34], and the stochastic epidemic models can provide an additional degree of realism if compared to their deterministic counterparts [10, 35-47]. In Particular, Mao et al. [26] obtained the interesting and surprising conclusion: even a sufficiently small noise can suppress explosions in population dynamics. Beretta et al. [35] obtained the stability of epidemic model with stochastic time delays influenced by probability under certain conditions. Carletti [36] studied the stable properties of a stochastic model for phage-bacteria interaction in open marine environment analytically and numerically. In [37], establishing some stochastic models and studying of several endemic infections with demography, Nåsell found that some deterministic models are unacceptable approximations of the stochastic models for a large range of realistic parameter values. Dalal et al. [39, 40] showed that stochastic models had nonnegative solutions and carried out analysis on the asymptotic stability of models. In [41], Yu et al. presented stochastic asymptotic stability of the epidemic point of the two-group SIR model with random perturbation. It is shown in [45] that the SIR model has a unique global positive and asymptotic solution. But to our knowledge, the research on the stochastic dynamics of the epidemic model with ratiodependent nonlinear incidence rate seems rare.

There are different possible approaches to including random effects in the model, both from a biological and from a mathematical perspectives [48]. Our basic approach is analogous to that of Beddington and May [20], which is pursued in [48], and also, for example, in $[45,47]$ to epidemic models, in which they considered that the environmental noise was proportional to the variables. Following them, in this paper, we assume that stochastic perturbations are of a white noise type which is directly proportional to $x(t), y(t)$, influenced on the $d x(t) / d t$ and $d y(t) / d t$ in model (4). In this way, we introduce stochastic perturbation terms into the growth equations of susceptible and infected individuals to incorporate the effect of randomly fluctuating environment, and the following stochastic differential equation is corresponding to model (7):

$$
\begin{gathered}
d x=\left(1-q x-y-\frac{a x y}{x+p y}\right) d t+\sigma_{1} x d B_{1}(t), \\
d y=\left(\frac{R_{0} x y}{x+p y}-y\right) d t+\sigma_{2} y d B_{2}(t),
\end{gathered}
$$

where $\sigma_{1}, \sigma_{2}$ are real constants and known as the intensity of environmental fluctuations, and $B_{1}(t), B_{2}(t)$ are independent standard Brownian motions. 
The aim of this paper is to consider the dynamics of the epidemic models (7) and (8). The paper is organized as follows. In Section 2, we give some properties about deterministic model (7). In Section 3, we carry out the analysis of the dynamical properties of stochastic model (8). And in Section 4, we give some numerical examples and make a comparative analysis of the stability of the model with deterministic and stochastic environments and have some discussions.

\section{Dynamics of the Deterministic Model}

Let us begin to determine the location and number of the equilibria of model (7). It is easy to see that if $R_{0}<1$, the disease-free point $E_{0}=(1 / q, 0)$ is the unique equilibrium, corresponding to the extinction of the disease; if $R_{0}>1$, in addition to the disease-free point $E_{0}$, there is a unique endemic point $E^{*}=\left(x^{*}, y^{*}\right)$, corresponding to the survival of the disease, described by the following expressions:

$$
x^{*}=\frac{p R_{0}}{p q R_{0}+\left(R_{0}+a\right)\left(R_{0}-1\right)}, \quad y^{*}=\frac{R_{0}-1}{p} x^{*} .
$$

The Jacobian matrix of model (7) at $E_{0}$ is as follows:

$$
\left(\begin{array}{cc}
-q & -1-a \\
0 & R_{0}-1
\end{array}\right)
$$

It follows that $E_{0}$ is asymptotically stable if $R_{0}<1$ and unstable if $R_{0}>1$.

The Jacobian matrix of model (7) at $E^{*}$ is as follows:

$$
J^{*}=\left(\begin{array}{ll}
J_{11} & J_{12} \\
J_{21} & J_{22}
\end{array}\right)
$$

where

$$
\begin{gathered}
J_{11}=-\frac{p q R_{0}^{2}+a\left(R_{0}-1\right)^{2}}{p R_{0}^{2}}, \quad J_{12}=-\frac{R_{0}^{2}+a}{R_{0}^{2}}, \\
J_{21}=\frac{\left(R_{0}-1\right)^{2}}{p R_{0}}, \quad J_{22}=-\frac{R_{0}-1}{R_{0}} .
\end{gathered}
$$

It is easy, by simple computations, to see that

$$
\begin{gathered}
\operatorname{tr}\left(J^{*}\right)=J_{11}+J_{22}<0 \\
\operatorname{det}\left(J^{*}\right)=\frac{p q R_{0}+\left(a+R_{0}\right)\left(R_{0}-1\right)}{p R_{0}^{2}}>0 .
\end{gathered}
$$

Summarizing the above, we have the following results on the dynamics of model (7).

Theorem 1. (i) If $R_{0}<1$, then model (7) has a unique diseasefree equilibrium $E_{0}$ which is asymptotically stable.

(ii) If $R_{0}>1$, then model (7) has two equilibria, a diseasefree equilibrium $E_{0}$ which is an unstable saddle and an endemic equilibrium $E^{*}$ which is asymptotically stable.

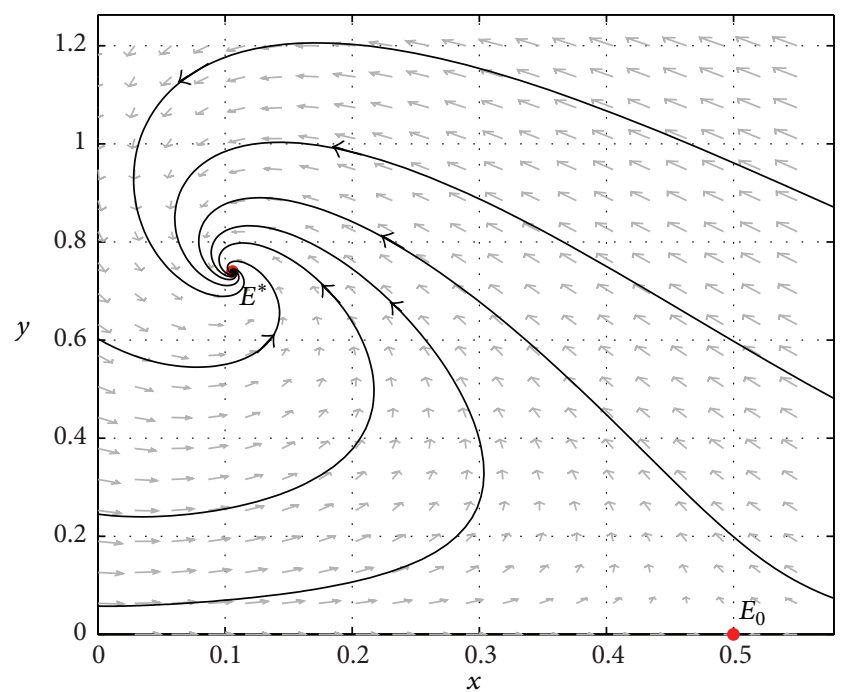

FIgURE 1: The dynamics of model (7). The parameters are taken as (14). $E_{0}=(0.5,0)$ is a saddle point. $E^{*}=(0.11,0.74)$ is globally asymptotically stable.

As a matter of fact, we can prove that the endemic point $E^{*}=\left(x^{*}, y^{*}\right)$ is also global asymptotically stable. For more details, see [19].

In Figure 1, we show the dynamics of the deterministic model (7) with the following parameters:

$$
a=0.3, \quad p=0.5, \quad q=2, \quad R_{0}=4.5 .
$$

In this case, $E_{0}=(0.5,0)$ is a saddle point. $E^{*}=(0.10563$, 0.73944 ) is globally asymptotically stable.

In the following, we will focus on the boundedness, dissipation, and persistence of mode (7).

Theorem 2. All the solutions of model (7) with the positive initial condition $(x(0), y(0))$ are uniformly bounded within a region $\Gamma$, where

$$
\Gamma=\left\{(x, y) \in \mathbb{R}_{+}^{2}: x+\frac{a}{R_{0}} y \leq \min \left\{\frac{1}{q}, \frac{R_{0}}{R_{0}+a}\right\}\right\} .
$$

Proof. Define function

$$
N(t)=x(t)+\frac{a}{R_{0}} y(t) .
$$

Differentiating $N(t)$ with respect to time $t$ along the solutions of model (7), we can get the following:

$$
\frac{d N(t)}{d t}=\frac{d x}{d t}+\frac{a}{R_{0}} \frac{d y}{d t}=1-q x-\left(1+\frac{a}{R_{0}}\right) y .
$$

Thus, we obtain the following:

$$
\frac{d N(t)}{d t}+\eta N(t)=1-(q-\eta) x-\left(1+\frac{a}{R_{0}}-\eta\right) y<1,
$$


where $\eta<\min \left\{q, 1+\left(a / R_{0}\right)\right\}$. And we obtain the following:

$$
0<N(x, y) \leq \frac{1}{\eta}+N(x(0), y(0)) e^{-\eta t} .
$$

As $t \rightarrow \infty, 0<N \leq 1 / \eta$. Therefore, all solutions of model (7) enter into the region $\Gamma$. This completes the proof.

Theorem 3. If $R_{0}>1$, model (7) is dissipative.

Proof. Since all solutions of model (7) are positive, by the first equation of (7), we have the following:

$$
\frac{d x}{d t} \leq 1-q x
$$

A standard comparison theorem shows that

$$
\limsup _{t \rightarrow \infty} x(t) \leq \frac{1}{q} .
$$

Hence, for any $0<\varepsilon \ll 1$ and large $t, x \leq(1 / q)+\varepsilon$. It then follows that $y$ satisfies the following:

$$
\frac{d y}{d t} \leq \frac{y\left(\left(\left(R_{0}-1\right) / q\right)+\varepsilon\left(R_{0}-1\right)-p y\right)}{(1 / q)+\varepsilon+p y} .
$$

The arbitrariness of $\varepsilon$ then implies that

$$
\limsup _{t \rightarrow \infty} y(t) \leq \frac{R_{0}-1}{p q} .
$$

Theorem 4. If $R_{0}>1$ and $p q<(1+a)\left(R_{0}-1\right)$, then model (7) is permanent; that is, there exists $\varepsilon>0$ (independent of initial conditions), such that $\liminf _{t \rightarrow \infty} x(t)>\varepsilon, \liminf _{t \rightarrow \infty} y(t)>$ $\varepsilon$.

Proof. By the first equation in (7), we have the following:

$$
\begin{aligned}
\frac{d x}{d t} & =1-q x-(1+a) y+\frac{a p y^{2}}{x+p y} \\
& >1-q x-(1+a) y .
\end{aligned}
$$

If $R_{0}>1$ and $p q<(1+a)\left(R_{0}-1\right)$, from the proof of Theorem 3, we see that $\lim \sup _{t \rightarrow \infty} y(t) \leq\left(R_{0}-1\right) / p q$. Thus, for any $0<\varepsilon \leq\left(R_{0}-1\right) / p q$ and large $t, y(t)>\left(\left(R_{0}-1\right) / p q\right)-\varepsilon$. As a result, we have the following:

$$
\frac{d x}{d t}>1-(1+a)\left(\frac{R_{0}-1}{p q}-\varepsilon\right)-q x .
$$

With the comparison principle, the arbitrariness of $\varepsilon$ implies that

$$
\liminf _{t \rightarrow \infty} x(t) \geq \frac{p q-(1+a)\left(R_{0}-1\right)}{p q^{2}} \triangleq \underline{x} .
$$

Hence, for any $0<\varepsilon<\left(p q-(1+a)\left(R_{0}-1\right)\right) / p q^{2}$ and large $t, x(t)>\underline{x}-\varepsilon$.
And for large $t$, we have the following:

$$
\frac{d y}{d t}>\frac{y\left((\underline{x}-\varepsilon)\left(R_{0}-1\right)-p y\right)}{\underline{x}-\varepsilon+p y} .
$$

Therefore,

$$
\liminf _{t \rightarrow \infty} x(t) \geq \frac{(\underline{x}-\varepsilon)\left(R_{0}-1\right)}{p} .
$$

The arbitrariness of $\varepsilon$ then implies that

$$
\liminf _{t \rightarrow \infty} x(t) \geq \frac{\underline{x}\left(R_{0}-1\right)}{p} \triangleq \underline{y} .
$$

Choosing a positive number $\epsilon$ such that $\epsilon<\min \{\underline{x} / 2, \underline{y} / 2\}$, we see that

$$
\liminf _{t \rightarrow \infty} x(t)>\epsilon, \quad \liminf _{t \rightarrow \infty} y(t)>\varepsilon .
$$

This ends the proof.

Noting that if the parameters of model (7) are fixed as (14), we can obtain the following:

$$
R_{0}>1, \quad p q=0.6<(1+a)\left(R_{0}-1\right)=4.55,
$$

and from Theorems 3 and 4, we can conclude that model (7) is dissipation and persistence.

\section{Dynamics of the Stochastic Model}

In this subsection, we investigate the dynamical behavior of the stochastic model (8). Throughout this paper, let $(\Omega, \mathscr{F}, \mathscr{P})$ be a complete probability space with a filtration $\left\{\mathscr{F}_{t}\right\}_{t \in \mathbb{R}_{+}}$ satisfying the usual conditions (i.e., it is right continuous and increasing while $\mathscr{F}_{0}$ contains all $\mathscr{P}$-null sets). $B_{1}(t), B_{2}(t)$ are the Brownian motions defined on this probability space. We denote by $X(t)=\left((x(t), y(t))\right.$ and $|X(t)|=\left(x^{2}(t)+y^{2}(t)\right)^{1 / 2}$. Denote $\Lambda=\left\{(x, y) \in \mathbb{R}_{+}^{2}: x \geq a / R_{0}, y>0\right\}$.

Denote by $C^{2,1}\left(\mathbb{R}^{d} \times(0, \infty) ; \mathbb{R}_{+}\right)$the family of all nonnegative functions $V(x, t)$ defined on $\mathbb{R}^{d} \times(0, \infty)$ such that they are continuously twice differentiable in $x$ and once in $t$. Define the differential operator $L$ associated with $d$ dimensional stochastic differential equation:

$$
d x(t)=f(x(t), t) d t+h(x(t), t) d B(t)
$$

by

$$
\begin{aligned}
L= & \frac{\partial}{\partial t}+\sum_{i=1}^{d} f_{i}(x, t) \frac{\partial}{\partial x_{i}} \\
& +\frac{1}{2} \sum_{i, j=1}^{d}\left[h^{T}(x, t) h(x, t)\right]_{i j} \frac{\partial^{2}}{\partial x_{i} \partial x_{j}} .
\end{aligned}
$$

If $L$ acts in a function $V \in C^{2,1}\left(\mathbb{R}^{d} \times(0, \infty) ; \mathbb{R}_{+}\right)$, then

$$
\begin{aligned}
L V(x, t)= & V_{t}(x, t)+V_{x}(x, t) f(x, t) \\
& +\frac{1}{2} \operatorname{trace}\left[h^{T}(x, t) V_{x x}(x, t) h(x, t)\right],
\end{aligned}
$$

where $T$ means transposition. 
3.1. Existence and Uniqueness of Global Positive Solutions. To investigate the dynamical behavior of model (8), the first thing concerned is whether the solution is global existent. In this section, using the Lyapunov analysis method (mentioned in [24]), we will show the solution of model (8) is global and nonnegative.

Lemma 5. There is a unique local positive solution $(x(t), y(t))$ for $t \in\left[0, \tau_{e}\right)$ to model (8) almost surely (a.s.) for the initial value $(x(0), y(0)) \in \Lambda$, where $\tau_{e}$ is the explosion time.

Proof. Set

$$
u(t)=\ln x(t), \quad v(t)=\ln y(t),
$$

by Itô formula, we have the following:

$$
\begin{gathered}
d u=\left(\frac{1}{e^{u}}-q-\frac{e^{v}}{e^{u}}-\frac{a e^{v}}{e^{u}+p e^{v}}-\frac{\sigma_{1}^{2}}{2}\right) d t+\sigma_{1} d B_{1}(t), \\
d v=\left(\frac{R_{0} e^{u}}{e^{u}+p e^{v}}-1-\frac{\sigma_{2}^{2}}{2}\right) d t+\sigma_{2} d B_{2}(t),
\end{gathered}
$$

at $t \geq 0$ with initial value $u(0)=\ln x(0), v(0)=\ln y(0)$.

It is easy to see that the coefficients of model (36) satisfy the local Lipschitz condition, and there is a unique local solution $u(t), v(t)$ on $\left[0, \tau_{e}\right)[24]$. Therefore, $x(t)=e^{u(t)}$, $y(t)=e^{v(t)}$ are the unique positive local solutions to model (36) with the initial value $(x(0), y(0)) \in \Lambda$.

Lemma 5 only tells us that there exists a unique local positive solution to model (8). In the following, we show this solution is global; that is, $\tau_{e}=\infty$, which is motived by the work of Luo and Mao [29].

Theorem 6. Consider model (8), for any given initial value $(x(0), y(0)) \in \Lambda$, there is a unique solution $(x(t), y(t))$ on $t \geq 0$ and the solution will remain in $\Lambda$ with probability 1.

Proof. Let $n_{0}>0$ be sufficiently large for $x(0)$ and $y(0)$ lying within the interval $\left[1 / n_{0}, n_{0}\right]$. For each integer $n>n_{0}$, define the stopping times:

$$
\tau_{n}=\inf \left\{t \in\left[0, \tau_{e}\right]: x(t) \notin\left(\frac{1}{n}, n\right) \text { or } y(t) \notin\left(\frac{1}{n}, n\right)\right\} .
$$

We set inf $\emptyset=\infty$ ( $\emptyset$ represents the empty set) in this paper. $\tau_{n}$ is increasing as $n \rightarrow \infty$. Let $\tau_{\infty}=\lim _{n \rightarrow \infty} \tau_{n}$; then $\tau_{\infty} \leq \tau_{e}$ a.s..

In the following, we need to show $\tau_{\infty}=\infty$ a.s. If this statement is violated, there exist constants $T>0$ and $\varepsilon \in(0,1)$ such that $\mathscr{P}\left\{\tau_{\infty} \leq T\right\}>\varepsilon$. As a consequence, there exists an integer $n_{1} \geq n_{0}$ such that

$$
\mathscr{P}\left\{\tau_{n} \leq T\right\} \geq \varepsilon, \quad n \geq n_{1} .
$$

Define a function $V_{1}: \Lambda \rightarrow \mathbb{R}_{+}$by the following:

$$
V_{1}(x, y)=\left(\frac{R_{0}}{a} x-1-\ln \frac{R_{0}}{a} x\right)+(y-1-\ln y),
$$

which is a non-negativity function.

If $(x(t), y(t)) \in \Lambda$, by the Itô formula, we compute the following:

$$
\begin{aligned}
d V_{1}= & {\left[\left(\frac{R_{0}}{a}-\frac{1}{x}\right)\left(1-q x-y-\frac{a x y}{x+p y}\right)+\frac{\sigma_{1}^{2}}{2}\right] d t } \\
& +\sigma_{1}\left(\frac{R_{0}}{a} x-1\right) d B_{1}(t) \\
& +\left[\left(1-\frac{1}{y}\right)\left(\frac{R_{0} x}{x+p y}-1\right) y+\frac{\sigma_{2}^{2}}{2}\right] d t \\
& +\sigma_{2}(y-1) d B_{2}(t) \\
= & L V_{1} d t+\sigma_{1}\left(\frac{R_{0}}{a} x-1\right) d B_{1}(t) \\
& +\sigma_{2}(y-1) d B_{2}(t),
\end{aligned}
$$

where

$$
\begin{aligned}
L V_{1}= & q+\frac{a y-R_{0} x}{x+p y}-\frac{R_{0} q}{a} x \\
& +\left(\frac{R_{0}}{a}-\frac{1}{x}\right)(1-y)+1-y+\frac{\sigma_{1}^{2}}{2}+\frac{\sigma_{2}^{2}}{2} \\
\leq & q+\frac{a}{p}+\left(\frac{R_{0}}{a}-\frac{1}{x}\right)(1-y) \\
& +1-y+\frac{\sigma_{1}^{2}}{2}+\frac{\sigma_{2}^{2}}{2} .
\end{aligned}
$$

Case 1 (assume $a \geq R_{0}$ ). In this case, we have $x \geq 1$. It follows that

$$
\begin{aligned}
\left(\frac{R_{0}}{a}\right. & \left.-\frac{1}{x}\right)(1-y)+1-y \\
& \leq \frac{R_{0}}{a}+y\left(\frac{1}{x}-1\right)-\frac{1}{x}+1 \leq 1+\frac{R_{0}}{a} .
\end{aligned}
$$

Case 2 (assume $a<R_{0}$ ). If $a / R_{0} \leq x<1$, one has the following:

(a) $\left(\left(R_{0} / a\right)-(1 / x)\right)(1-y)+1-y \leq\left(R_{0} / a\right)+((y-1) / x)+$ $1-y \leq 1+\left(R_{0} / a\right)$ provided that $0<y \leq 1$;

(b) $\left(\left(R_{0} / a\right)-(1 / x)\right)(1-y)+1-y \leq 1$ provided that $y>1$.

Hence, there exists a positive number $M$ independent on $x, y$ and $t$ such that $L V_{1} \leq M$. Substituting this inequality into (40), we can get the following:

$$
\begin{aligned}
d V_{1} \leq & M d t+\sigma_{1}\left(\frac{R_{0}}{a} x-1\right) d B_{1}(t) \\
& +\sigma_{2}(y-1) d B_{2}(t) .
\end{aligned}
$$

Integrating both sides of the above inequality from 0 to $\tau_{n} \wedge T$ and taking expectations leads to the following:

$$
E V_{1}\left(x\left(\tau_{n} \wedge T\right), y\left(\tau_{n} \wedge T\right)\right) \leq V_{1}(x(0), y(0))+M T .
$$


Set $\Omega_{n}=\left\{\tau_{n} \leq T\right\}$, for $n \geq n_{1}$ and consider inequality (38), we can get $\mathscr{P}\left(\Omega_{n}\right) \geq \varepsilon$. Note that for every $\omega \in \Omega_{n}$, there exists some $i$ such that $x_{i}\left(\tau_{n}, \omega\right)$ equals either $n$ or $1 / n$ for $i=1,2$; hence,

$$
\begin{aligned}
& V_{1}\left(x\left(\tau_{n}, \omega\right), y\left(\tau_{n}, \omega\right)\right) \\
& \quad \geq \min \left\{(n-1-\ln n),\left(\frac{1}{n}-1-\ln \frac{1}{n}\right)\right\} .
\end{aligned}
$$

It then follows from (44) that

$$
\begin{aligned}
& V_{1}(x(0), y(0))+M T \\
& \quad \geq E\left[I_{\Omega_{n}(\omega)} V_{1}\left(x\left(\tau_{n}\right), y\left(\tau_{n}\right)\right)\right] \\
& \quad \geq \epsilon \min \left\{(n-1-\ln n),\left(\frac{1}{n}-1-\ln \frac{1}{n}\right)\right\},
\end{aligned}
$$

where $I_{\Omega_{n}}$ is the indicator function of $\Omega_{n}$.

As $n \rightarrow \infty$ we have the following:

$$
\infty>V_{1}(x(0), y(0))+M T=\infty \quad \text { a.s., }
$$

which leads to the contradiction. This completes the proof.

3.2. Stochastic Boundedness and Permanence. Theorem 6 shows that the solutions to model (8) will remain in $\Lambda$. Generally speaking, the nonexplosion property, the existence, and the uniqueness of the solution are not enough but the property of boundedness and permanence are more desirable since they mean the long-time survival in the population dynamics. Now, we present the definition of stochastic ultimate boundedness and stochastic permanence [31].

Definition 7. The solutions $X(t)=(x(t), y(t))$ of model (8) are said to be stochastically ultimately bounded, if for any $\varepsilon \epsilon$ $(0,1)$, there is a positive constant $\delta=\delta(\varepsilon)$, such that for any initial value $(x(0), y(0)) \in \Lambda$, the solution $X(t)$ of model (8) has the property that

$$
\limsup _{t \rightarrow \infty} P\{|X(t)|>\delta\}<\varepsilon .
$$

Definition 8. The solutions $X(t)=(x(t), y(t))$ of model (8) are said to be stochastically permanent if for any $\varepsilon \in(0,1)$, there exists a pair of positive constants $\delta=\delta(\varepsilon)$ and $\chi=\chi(\varepsilon)$, such that for any initial value $(x(0), y(0)) \in \Lambda$, the solution $X(t)$ of model (8) has the property that

$$
\begin{aligned}
& \liminf _{t \rightarrow \infty} P\{|X(t)| \geq \delta\} \geq 1-\varepsilon, \\
& \liminf _{t \rightarrow \infty} P\{|X(t)| \leq \chi\} \geq 1-\varepsilon .
\end{aligned}
$$

Theorem 9. The solutions of model (8) are stochastically ultimately bounded for any initial value $(x(0), y(0)) \in \Lambda$.

Proof. Denote functions

$$
V_{2}=e^{t} x^{\theta}, \quad V_{3}=e^{t} y^{\theta}
$$

for $(x, y) \in \Lambda$ and $0<\theta<1$.
Applying the Itô formula leads to the following:

$$
\begin{aligned}
& d V_{2}=L V_{2} d t+\sigma_{1} \theta e^{t} x^{\theta} d B_{1}(t), \\
& d V_{3}=L V_{3} d t+\sigma_{2} \theta e^{t} y^{\theta} d B_{2}(t),
\end{aligned}
$$

where

$$
\begin{gathered}
L V_{2}=e^{t} x^{\theta}\left(1+\theta\left(\frac{1}{x}-q-\frac{y}{x}-\frac{a y}{x+p y}\right)+\frac{\sigma_{1}^{2} \theta(\theta-1)}{2}\right), \\
L V_{3}=e^{t} y^{\theta}\left(1+\theta\left(\frac{R_{0} x}{x+p y}-1\right)+\frac{\sigma_{2}^{2} \theta(\theta-1)}{2}\right) .
\end{gathered}
$$

Thus, there exists the positive constants $M_{1}$ and $M_{2}$ such that we have $L V_{2}<M_{1} e^{t}$ and $L V_{3}<M_{2} e^{t}$. It follows that $e^{t} E x^{\theta}-$ $E x(0)^{\theta} \leq M_{1} e^{t}$ and $e^{t} E y^{\theta}-E y(0)^{\theta} \leq M_{2} e^{t}$. Then we get the following:

$$
\begin{aligned}
& \limsup _{t \rightarrow \infty} E x^{\theta} \leq M_{1}<+\infty, \\
& \limsup _{t \rightarrow \infty} E y^{\theta} \leq M_{2}<+\infty .
\end{aligned}
$$

Note that

$$
\begin{aligned}
|X(t)|^{\theta} & =\left(x^{2}(t)+y^{2}(t)\right)^{\theta / 2} \\
& \leq 2^{\theta / 2} \max \left\{x^{\theta}(t), y^{\theta}(t)\right\} \\
& \leq 2^{\theta / 2}\left(x^{\theta}+y^{\theta}\right) .
\end{aligned}
$$

Therefore, we obtain the following:

$$
\limsup _{t \rightarrow \infty} E|X(t)|^{\theta} \leq 2^{\theta / 2}\left(M_{1}+M_{2}\right)<+\infty .
$$

As a result, there exists a positive constant $\delta_{1}$ such that

$$
\limsup _{t \rightarrow \infty} E(\sqrt{|X(t)|})<\delta_{1} .
$$

Now, for any $\varepsilon>0$, let $\delta=\delta_{1}^{2} / \varepsilon^{2}$; then by Chebyshev's inequality,

$$
\mathscr{P}\{|X(t)|>\delta\} \leq \frac{E(\sqrt{|X(t)|})}{\sqrt{\delta}} .
$$

Hence,

$$
\limsup _{t \rightarrow \infty} \mathscr{P}\{|X(t)|>\delta\} \leq \frac{\delta_{1}}{\sqrt{\delta}}=\varepsilon,
$$

which yields the required assertion.

We are now in the position to show the stochastic permanence. Let us present some hypothesis and a useful lemma. 
Lemma 10. Assume $R_{0}>a+\max \{4,2 p q\}$. For any initial value $(x(0), y(0)) \in \Lambda$, the solution $(x(t), y(t))$ satisfies that

$$
\limsup _{t \rightarrow \infty} E\left(\frac{1}{|X(t)|^{\rho}}\right) \leq H,
$$

where $\rho$ is an arbitrary positive constant satisfying

$$
\begin{aligned}
& \frac{\rho+1}{2}\left(\max \left\{\sigma_{1}, \sigma_{2}\right\}\right)^{2}<1+\min \left\{\frac{R_{0}-a}{2 p}-q, \frac{R_{0}-a}{2}-2\right\}, \\
& H=\frac{2^{\rho}\left(C_{2}+4 k C_{1}\right)}{4 k C_{1}} \\
& \quad \times \max \left\{1,\left(\frac{2 C_{1}+C_{2}+\sqrt{C_{2}^{2}+4 C_{1} C_{2}}}{2 C_{1}}\right)^{\rho-2}\right\}
\end{aligned}
$$

in which $k$ is an arbitrary positive constant satisfying

$$
\begin{aligned}
& \frac{\rho(\rho+1)}{2}\left(\max \left\{\sigma_{1}, \sigma_{2}\right\}\right)^{2}+k \\
& <\rho+\rho \min \left\{\frac{R_{0}-a}{2 p}-q, \frac{R_{0}-a}{2}-2\right\}
\end{aligned}
$$

with

$$
\begin{aligned}
C_{1}= & \rho+\rho \min \left\{\frac{R_{0}-a}{2 p}-q, \frac{R_{0}-a}{2}-2\right\} \\
& -\frac{\rho(\rho+1)}{2}\left(\max \left\{\sigma_{1}, \sigma_{2}\right\}\right)^{2}-k>0, \\
C_{2}= & \rho \max \{q, 2+a\}+\frac{\rho R_{0}\left(R_{0}-1\right) \max \left\{1, p^{2}\right\}}{2 a p} \\
& +\rho\left(\max \left\{\sigma_{1}, \sigma_{2}\right\}\right)^{2}+2 k>0 .
\end{aligned}
$$

Proof. Set $U(x, y)=1 /(x+y)$ for $(x(t), y(y)) \in \Lambda$, by the Itô formula, we have the following:

$$
\begin{aligned}
d U= & -U^{2}\left[1-q x-y-\frac{a x y}{x+p y}+\frac{R_{0} x y}{x+p y}-y\right] d t \\
& +U^{3}\left(\sigma_{1}^{2} x^{2}+\sigma_{2}^{2} y^{2}\right) d t \\
& -U^{2}\left(\sigma_{1} x d B_{1}(t)+\sigma_{2} y d B_{2}(t)\right) \\
= & L U d t-U^{2}\left(\sigma_{1} x d B_{1}(t)+\sigma_{2} y d B_{2}(t)\right),
\end{aligned}
$$

where

$$
\begin{aligned}
L U= & -U^{2}\left(1-q x-2 y+\frac{\left(R_{0}-a\right) x y}{x+p y}\right) \\
& +U^{3}\left(\sigma_{1}^{2} x^{2}+\sigma_{2}^{2} y^{2}\right) .
\end{aligned}
$$

Choose a positive constant $\rho$ such that it satisfies (60). Applying the Itô formula again, we can get the following:

$$
\begin{aligned}
L[ & \left.(1+U)^{\rho}\right] \\
= & \rho(1+U)^{\rho-1} L U \\
& \quad+\frac{\rho(\rho-1)}{2} U^{4}(1+U)^{\rho-2}\left(\sigma_{1}^{2} x^{2}+\sigma_{2}^{2} y^{2}\right) \\
& =(1+U)^{\rho-2} \Phi,
\end{aligned}
$$

where

$$
\begin{aligned}
\Phi= & -\rho U^{2}\left(1-q x-2 y+\frac{\left(R_{0}-a\right) x y}{x+p y}\right) \\
& -\rho U^{3}\left(1-q x-2 y+\frac{\left(R_{0}-a\right) x y}{x+p y}\right) \\
& +\rho U^{3}\left(\sigma_{1}^{2} x^{2}+\sigma_{2}^{2} y^{2}\right) \\
& +\frac{\rho(1+\rho) U^{4}}{2}\left(\sigma_{1}^{2} x^{2}+\sigma_{2}^{2} y^{2}\right) \\
\leq & -\rho U^{2}+\rho U^{2}(q x+(2+a) y) \\
& -\rho U^{3}\left(\left(\frac{R_{0}-a}{2 p}-q\right) x+\left(\frac{R_{0}-a}{2}-2\right) y\right) \\
& +\rho U^{3}\left(\frac{\left(R_{0}-a\right)\left(x^{2}+p^{2} y^{2}\right)}{2 p(x+p y)}\right) \\
& +\rho U^{3}\left(\sigma_{1}^{2} x^{2}+\sigma_{2}^{2} y^{2}\right) \\
& +\frac{\rho(1+\rho) U^{4}}{2}\left(\sigma_{1}^{2} x^{2}+\sigma_{2}^{2} y^{2}\right) .
\end{aligned}
$$

Using the facts that

$$
\begin{aligned}
U^{3}\left(\sigma_{1}^{2} x^{2}+\sigma_{2}^{2} y^{2}\right) & <\left(\max \left\{\sigma_{1}, \sigma_{2}\right\}\right)^{2} U, \\
U^{4}\left(\sigma_{1}^{2} x^{2}+\sigma_{2}^{2} y^{2}\right) & <\left(\max \left\{\sigma_{1}, \sigma_{2}\right\}\right)^{2} U^{2},
\end{aligned}
$$

so,

$$
\begin{aligned}
\Phi \leq- & U^{2}\left(\rho+\rho \min \left\{\frac{R_{0}-a}{2 p}-q, \frac{R_{0}-a}{2}-2\right\}\right. \\
& \left.-\frac{\rho(\rho+1)}{2}\left(\max \left\{\sigma_{1}, \sigma_{2}\right\}\right)^{2}\right) \\
+ & U\left(\rho \max \{q, 2+a\}+\frac{\rho R_{0}\left(R_{0}-1\right) \max \left\{1, p^{2}\right\}}{2 a p}\right. \\
& \left.+\rho\left(\max \left\{\sigma_{1}, \sigma_{2}\right\}\right)^{2}\right) .
\end{aligned}
$$


Now, let $k>0$ sufficiently small such that it satisfies (62), by the Itô formula; then

$$
\begin{aligned}
& L\left[e^{k t}(1+U)^{\rho}\right] \\
& \quad=k e^{k t}(1+U)^{\rho}+e^{k t} L(1+U)^{\rho} \\
& \quad=e^{k t}(1+U)^{\rho-2}\left(k(1+U)^{2}+\Phi\right) \\
& \quad \leq e^{k t}(1+U)^{\rho-2}\left(-C_{1} U^{2}+C_{2} u+k\right) \\
& \quad \leq H_{1} e^{k t},
\end{aligned}
$$

where $H_{1}=\left(\left(C_{2}+4 k C_{1}\right) / 4 C_{1}\right) \max \left\{1,\left(\left(2 C_{1}+C_{2}+\right.\right.\right.$ $\left.\left.\left.\sqrt{C_{2}^{2}+4 C_{1} C_{2}}\right) / 2 C_{1}\right)^{\rho-2}\right\}$ and $C_{1}, C_{2}$ have been defined in the statement of the theorem. Thus,

$$
E\left[e^{k t}(1+U)^{\rho}\right] \leq(1+U(0))^{\rho}+\frac{H_{1}}{k} e^{k t} .
$$

So we can have the following:

$$
\limsup _{t \rightarrow \infty} E\left[U(t)^{\rho}\right] \leq \limsup _{t \rightarrow \infty} E(1+U)^{\rho} \leq \frac{H_{1}}{k} .
$$

In addition, we know that $(x+y)^{\rho} \leq 2^{\rho}\left(x^{2}+y^{2}\right)^{\rho / 2}=$ $2^{\rho}|X(t)|^{\rho}$; consequently,

$$
\begin{aligned}
\limsup _{t \rightarrow \infty} E\left[\frac{1}{|X(t)|^{\rho}}\right] & \leq 2^{\rho} \limsup _{t \rightarrow \infty} E\left[U(t)^{\rho}\right] \\
& \leq \frac{2^{\rho} H_{1}}{k}=H,
\end{aligned}
$$

which complets the proof.

Consider Chebyshev inequality, Theorem 9, and Lemma 10 together, we immediately obtain the following result.

Theorem 11. If the following conditions are satisfied

(i) $a+\max \{2 p q, 4\}<R_{0}$;

(ii) $(1 / 2)\left(\max \left\{\sigma_{1}, \sigma_{2}\right\}\right)^{2}<1+\min \left\{\left(\left(R_{0}-a\right) / 2 p\right)-q,\left(\left(R_{0}-\right.\right.\right.$ a)/2) -2$\}$,

then the solutions of model (8) is stochastically permanent.

\section{Conclusions and Discussions}

In this paper, by using the theory of stochastic differential equation, we investigate the dynamics of an SIRS epidemic model with a ratio-dependent incidence rate. The value of this study lies in two aspects. First, it presents some relevant properties of the deterministic model (7), including boundedness, dissipation, persistence, and the stability of the disease-free and endemic points. Second, it verifies the existence of global positive solutions, stochastic boundedness, and permanence for the stochastic model (8).

As an example, we give some numerical examples to illustrate the dynamical behavior of stochastic model (8) by using the Milstein method mentioned in Higham [49]. In this way, model (8) can be rewritten as the following discretization equations:

$$
\begin{aligned}
x_{k+1}= & x_{k}+\left(1-q x_{k}-y_{k}-\frac{a x_{k} y_{k}}{x_{k}+p y_{k}}\right) \Delta t \\
& +\sigma_{1} x_{k} \sqrt{\Delta t} \xi_{k}+\frac{\sigma_{1}^{2}}{2} x_{k}^{2}\left(\xi_{k}^{2}-1\right) \Delta t, \\
y_{k+1}= & y_{k}+\left(\frac{R_{0} x_{k} y_{k}}{x_{k}+p y_{k}}-y_{k}\right) \Delta t \\
& +\sigma_{2} y_{k} \sqrt{\Delta t} \eta_{k}+\frac{\sigma_{2}^{2}}{2} y_{k}^{2}\left(\eta_{k}^{2}-1\right) \Delta t,
\end{aligned}
$$

where $\xi_{k}$ and $\eta_{k}, k=1,2, \ldots, n$, are the Gaussian random variables $N(0,1)$.

The parameters of model (8) are fixed as (14). In this case, model $(7)$ has the endemic point $E^{*}=(0.11,0.74)$. And model (8) becomes as follows:

$$
\begin{gathered}
d x=\left(1-2 x-y-\frac{0.3 x y}{x+0.5 y}\right) d t+\sigma_{1} x d B_{1}(t), \\
d y=\left(\frac{4.5 x y}{x+0.5 y}-y\right) d t+\sigma_{2} y d B_{2}(t) .
\end{gathered}
$$

Simple computations show that

$$
\begin{gathered}
a+\max \{2 p q, 4\}=4.3<4.5=R_{0}, \\
\frac{0.03^{2}}{2}=\frac{1}{2}\left(\max \left\{\sigma_{1}, \sigma_{2}\right\}\right)^{2} \\
<1+\min \left\{\frac{R_{0}-a}{2 p}-q, \frac{R_{0}-a}{2}-2\right\}=1.1, \\
\left.\frac{0.5^{2}}{2}=\frac{1}{2}\left(\max \left\{\sigma_{1}, \sigma_{2}\right\}\right)^{2}, \sigma_{2}\right)=(0.03,0.01), \\
<1+\min \left\{\frac{R_{0}-a}{2 p}-q, \frac{R_{0}-a}{2}-2\right\}=1.1, \\
\text { if }\left(\sigma_{1}, \sigma_{2}\right)=(0.5,0.3) .
\end{gathered}
$$

It is easy to see that, all the conditions of Theorem 11 are satisfied, and we can therefore conclude that, with $\left(\sigma_{1}, \sigma_{2}\right)=$ $(0.03,0.01)$ and $\left(\sigma_{1}, \sigma_{2}\right)=(0.5,0.3)$, the solutions of model (8) is stochastically permanent. The numerical examples shown in Figures 2 and 3 clearly support these results. In Figure 2, with $\left(\sigma_{1}, \sigma_{2}\right)=(0.03,0.01)$, the solutions of model (8) will be oscillating slightly around the endemic point $E^{*}=$ $(0.11,0.74)$ of model $(7)$. And in Figure 3 , with $\left(\sigma_{1}, \sigma_{2}\right)=$ $(0.5,0.3)$, the solutions of model (8) will be oscillating strongly around the endemic point $E^{*}=(0.11,0.74)$ of model (7).

It is worthy to note that, throughout this paper, the parameters for model (7), also for model (8), are fixed as the set 


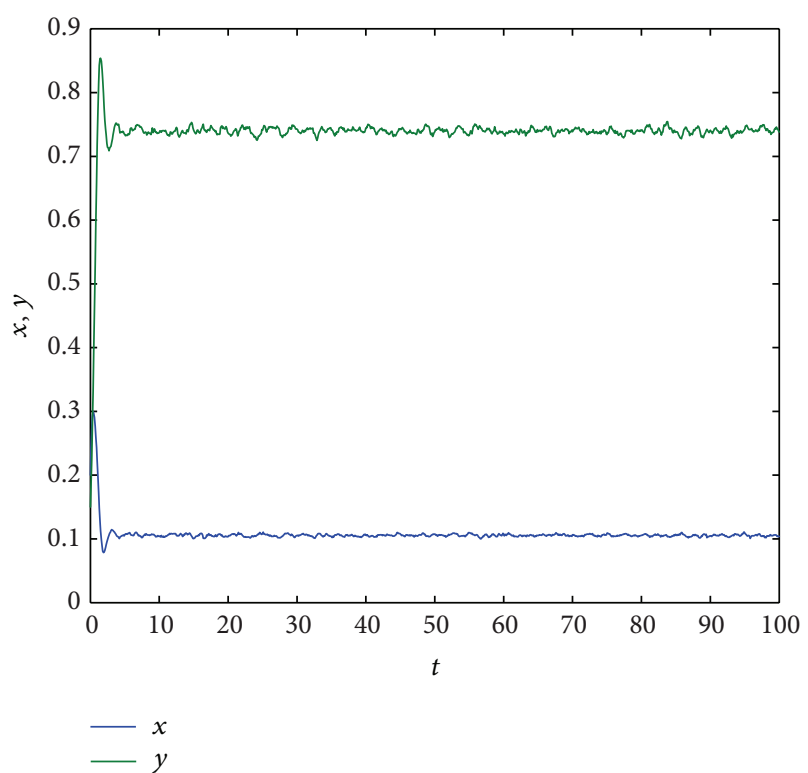

FIGURE 2: The solution of the stochastic model (8) with initial values $x(0)=0.2, y(0)=0.15$. The parameters are taken as (14), $\sigma_{1}=0.03$, $\sigma_{2}=0.01$.
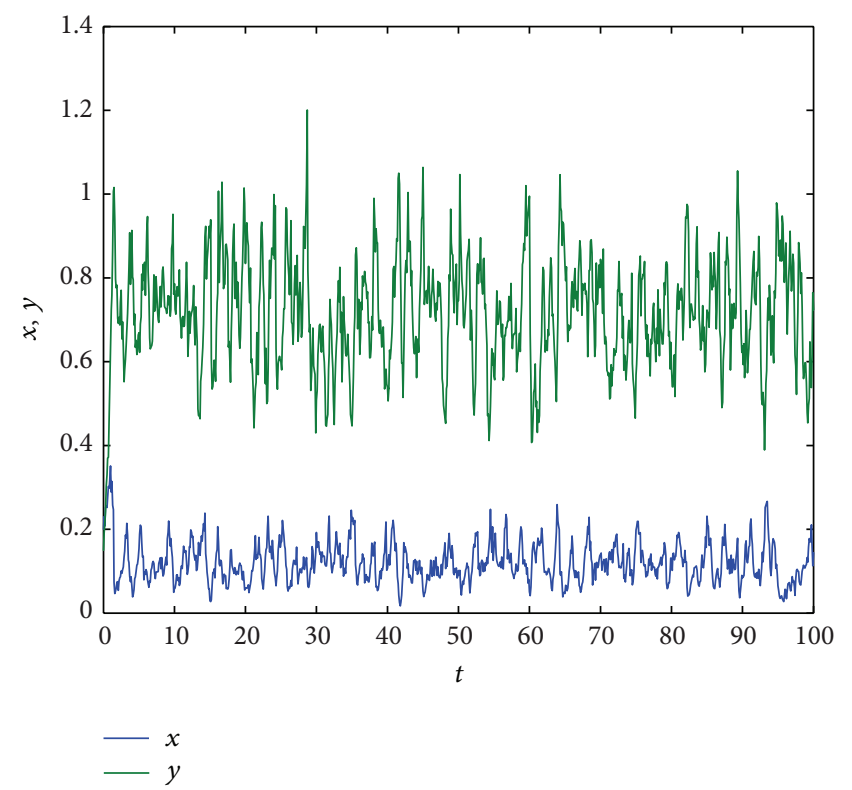

FIGURE 3: The solution of the stochastic model (8) with initial values $x(0)=0.2, y(0)=0.15$. The parameters are taken as $(14), \sigma_{1}=0.5$, $\sigma_{2}=0.3$.

(14). The reason is that with this parameter set, the conditions of our theoretical results hold. Of course, one can adopt other parameters set to show the numerical results.

From the theoretical and numerical results, we can know that, when the noise density is not large, the stochastic model (8) preserves the property of the stability of the deterministic model (7). To a great extent, we can ignore the noise and use the deterministic model (7) to describe the population dynamics. However, when the noise is sufficiently large, it can force the population to become largely fluctuating. In this case, we cannot use deterministic model (7) but stochastic model (8) to describe the population dynamics. Needless to say, both deterministic and stochastic epidemic models have their important roles.

Furthermore, from the numerical results in Figure 2, one can see that model (8) is stochastically stable. But we cannot prove the stochastic stability because of the complexity of model (8). This can be further investigated.

On the other hand, we know that there are different possible approaches to including random effects in the epidemic models affected by environmental white noise, here we consider another method to introduce random effects in the epidemic model (7). The martingale approach was initiated by Beretta et al. [35] and applied in [27, 30, 45, 47]. They introduced stochastic perturbation terms into the growth equations to incorporate the effect of a randomly fluctuating environment. In detail, assume that the stochastic perturbations of the state variables around their steady-state $E^{*}$ are of a white noise type which is proportional to the distances of $x, y$ from their steady-state values $x^{*}$ and $y^{*}$, respectively. In this way, model (7) will be reduced to the following form:

$$
\begin{aligned}
& d x=\left(1-q x-y-\frac{a x y}{x+p y}\right) d t+\sigma_{1}\left(x-x^{*}\right) d B_{1}(t), \\
& d y=\left(\frac{R_{0} x y}{x+p y}-y\right) d t+\sigma_{2}\left(y-y^{*}\right) d B_{2}(t),
\end{aligned}
$$

where the definitions of $\sigma_{1}, \sigma_{2}$ and $B_{1}(t), B_{2}(t)$ are the same as in (8).

If $R_{0}>1$, stochastic model (77) can center at its endemic point $E^{*}$, with the change of variables $u=x-x^{*}, v=y-y^{*}$. The linearized version of model (77) is as follows:

$$
d z(t)=f_{1}(z(t)) d t+f_{2}(z(t)) d B(t),
$$

where

$$
\begin{gathered}
z(t)=\left(\begin{array}{l}
u(t) \\
v(t)
\end{array}\right), \quad f_{1}=\left(\begin{array}{l}
J_{11} u(t)+J_{12} v(t) \\
J_{21} u(t)+J_{22} v(t)
\end{array}\right), \\
f_{2}=\left(\begin{array}{cc}
\sigma_{1} u(t) & 0 \\
0 & \sigma_{2} v(t)
\end{array}\right),
\end{gathered}
$$

where $J_{11}, J_{12}, J_{21}, J_{22}$ are defined as (12).

It is easy to see that the stability of the endemic point $E^{*}$ of model (77) is equivalent to the stability of zero solution of model (78).

Before proving the stochastic stability of the zero solution of model (78), we put forward a lemma in [50].

Lemma 12. Suppose there exists a function $V(z, t) \in C^{2}(\Omega)$ satisfying the following inequalities:

$$
\begin{gathered}
K_{1}|z|^{\omega} \leq V(z, t) \leq K_{2}|z|^{\omega}, \\
L V(z, t) \leq-K_{3}|z|^{\omega},
\end{gathered}
$$

where $\omega>0$ and $K_{i}(i=1,2,3)$ is positive constant. Then the zero solution of mode (78) is exponentially $\omega$-stable for all time $t \geq 0$. 
From the lemma above, note that if $\omega=2$ in (80) and (81), then the zero solution of model (78) is stochastically asymptotically stable in probability. Thus, we obtain the following theorem.

Theorem 13. Assume that $\sigma_{1}^{2}<2\left(p q R_{0}^{2}+a\left(R_{0}-1\right)^{2}\right) / p R_{0}^{2}$, $\sigma_{2}^{2}<2\left(R_{0}-1\right) / R_{0}$ hold; then the zero solution of model (78) is asymptotically mean square stable. And the endemic point $E^{*}$ of model (77) is asymptotically mean square stable.

The details of the proof are shown in the Appendix.

We should point out that the results obtained in this paper are only for the simple case when $l=h=1$ of the incidence rate (3). The dynamical behaviors of the stochastic epidemic model with general ratio-dependent incidence rate (3) are desirable in future studies.

\section{Appendix}

\section{The proof of Theorem 13}

Proof. Let us consider the Lyapunov function

$$
V_{5}(z(t))=\frac{1}{2}\left(u^{2}+\kappa v^{2}\right)
$$

where $\kappa=\left(R_{0}^{2}+a\right) / R_{0}\left(R_{0}-1\right)^{2}$.

It is easy to check that inequality (80) holds with $\omega=2$. Moreover,

$$
\begin{aligned}
L V_{5}(z(t))= & u\left(J_{11} u+J_{12} v\right)+\kappa v\left(J_{21} u+J_{22} v\right) \\
& +\frac{1}{2}\left(\sigma_{1}^{2} u^{2}+\kappa \sigma_{2}^{2} v^{2}\right) \\
= & \left(J_{11}+\frac{\sigma_{1}^{2}}{2}\right) u^{2}+\kappa\left(J_{22}+\frac{\sigma_{2}^{2}}{2}\right) v^{2} \\
= & -z^{T} \mathrm{Q} z,
\end{aligned}
$$

where

$$
Q=\left(\begin{array}{cc}
J_{11}+\frac{\sigma_{1}^{2}}{2} & 0 \\
0 & \kappa\left(J_{22}+\frac{\sigma_{2}^{2}}{2}\right)
\end{array}\right)
$$

When $\sigma_{1}^{2}<2\left(p q R_{0}^{2}+a\left(R_{0}-1\right)^{2}\right) / p R_{0}^{2}, \sigma_{2}^{2}<2\left(R_{0}-1\right) / R_{0}$, the two eigenvalues $\lambda_{1}, \lambda_{2}$ of the matrix $Q$ will be positive. Set $\lambda_{\text {min }}=\min \left\{\lambda_{1}, \lambda_{2}\right\}$, it follows from (A.2) immediately that

$$
L V_{5}(z(t)) \leq-\lambda_{\min }|z(t)|^{2} .
$$

We therefore have the assertion.

\section{Acknowledgments}

The authors would like to thank the editors and referees for their helpful comments and suggestions. They also thank Natural Science Foundation of Zhejiang Province (LY12A01014) and the National Basic Research Program of China (2012CB426510).

\section{References}

[1] W. O. Kermack and A. G. McKendrick, "Contributions to the mathematical theory of epidemics-I," Bulletin of Mathematical Biology, vol. 53, no. 1-2, pp. 33-55, 1991.

[2] Z. Ma, Y. Zhou, and J. Wu, Modeling and Dynamics of Infectious Diseases, Higher Education Press, Beijing, China, 2009.

[3] C. Bain, "Applied mathematical ecology," Journal of Epidemiology and Community Health, vol. 44, no. 3, p. 254, 1990.

[4] A. Korobeinikov and P. K. Maini, "Non-linear incidence and stability of infectious disease models," Mathematical Medicine and Biology, vol. 22, no. 2, pp. 113-128, 2005.

[5] J. Ma and Z. Ma, "Epidemic threshold conditions for seasonally forced SEIR models," Mathematical Biosciences and Engineering, vol. 3, no. 1, pp. 161-172, 2006.

[6] V. Capasso and G. Serio, "A generalization of the KermackMcKendrick deterministic epidemic model," Mathematical Biosciences, vol. 42, no. 1-2, pp. 43-61, 1978.

[7] V. Capasso, Mathematical Structures of Epidemic Systems, vol. 97, Springer, Berlin, Germany, 2008.

[8] W. M. Liu, S. A. Levin, and Y. Iwasa, "Influence of nonlinear incidence rates upon the behavior of SIRS epidemiological models," Journal of Mathematical Biology, vol. 23, no. 2, pp. 187204, 1986.

[9] W. M. Liu, H. W. Hethcote, and S. A. Levin, "Dynamical behavior of epidemiological models with nonlinear incidence rates," Journal of Mathematical Biology, vol. 25, no. 4, pp. 359-380, 1987.

[10] J. A. Jacquez and P. O'Neill, "Reproduction numbers and thresholds in stochastic epidemic models I. Homogeneous populations," Mathematical Biosciences, vol. 107, no. 2, pp. 161-186, 1991.

[11] W. R. Derrick and P. van den Driessche, "A disease transmission model in a nonconstant population," Journal of Mathematical Biology, vol. 31, no. 5, pp. 495-512, 1993.

[12] H. W. Hethcote, "The mathematics of infectious diseases," SIAM Review, vol. 42, no. 4, pp. 599-653, 2000.

[13] H. W. Hethcote and S. A. Levin, "Periodicity in epidemiological models," Applied Mathematical Ecology, vol. 18, pp. 193-211, 1989.

[14] M. E. Alexander and S. M. Moghadas, "Periodicity in an epidemic model with a generalized non-linear incidence," Mathematical Biosciences, vol. 189, no. 1, pp. 75-96, 2004.

[15] S. Ruan and W. Wang, "Dynamical behavior of an epidemic model with a nonlinear incidence rate," Journal of Differential Equations, vol. 188, no. 1, pp. 135-163, 2003.

[16] D. Xiao and S. Ruan, "Global analysis of an epidemic model with nonmonotone incidence rate," Mathematical Biosciences, vol. 208, no. 2, pp. 419-429, 2007.

[17] X.-Z. Li, W.-S. Li, and M. Ghosh, "Stability and bifurcation of an SIR epidemic model with nonlinear incidence and treatment," Applied Mathematics and Computation, vol. 210, no. 1, pp. 141$150,2009$.

[18] S. Yuan and B. Li, "Global dynamics of an epidemic model with a ratio-dependent nonlinear incidence rate," Discrete Dynamics in Nature and Society, vol. 2009, Article ID 609306, 13 pages, 2009.

[19] B. Li, S. Yuan, and W. Zhang, "Analysis on an epidemic model with a ratio-dependent nonlinear incidence rate," International Journal of Biomathematics, vol. 4, no. 2, pp. 227-239, 2011.

[20] J. R. Beddington and R. M. May, "Harvesting natural populations in a randomly fluctuating environment," Science, vol. 197, no. 4302 , pp. $463-465,1977$. 
[21] L. J. S. Allen, "An introduction to stochastic epidemic models," in Mathematical Epidemiology, F. Brauer, P. van den Driessche, and J. Wu, Eds., vol. 1945, pp. 81-130, Springer, Berlin, Germany, 2008.

[22] T. C. Gard, Introduction to Stochastic Differential Equations, vol. 114, Marcel Dekker, New York, NY, USA, 1988.

[23] B. Øksendal, Stochastic Differential Equations, Springer, Berlin, Germany, 4th edition, 1995.

[24] X. Mao, Stochastic Differential Equations and Their Applications, Horwood Publishing Limited,, Chichester, UK, 1997.

[25] R. Z. Khasminskii and F. C. Klebaner, "Long term behavior of solutions of the Lotka-Volterra system under small random perturbations," The Annals of Applied Probability, vol. 11, no. 3, pp. 952-963, 2001.

[26] X. Mao, G. Marion, and E. Renshaw, "Environmental Brownian noise suppresses explosions in population dynamics," Stochastic Processes and their Applications, vol. 97, no. 1, pp. 95-110, 2002.

[27] M. Bandyopadhyay and J. Chattopadhyay, "Ratio-dependent predator-prey model: effect of environmental fluctuation and stability," Nonlinearity, vol. 18, no. 2, pp. 913-936, 2005.

[28] D. Jiang and N. Shi, "A note on nonautonomous logistic equation with random perturbation," Journal of Mathematical Analysis and Applications, vol. 303, no. 1, pp. 164-172, 2005.

[29] Q. Luo and X. Mao, "Stochastic population dynamics under regime switching," Journal of Mathematical Analysis and Applications, vol. 334, no. 1, pp. 69-84, 2007.

[30] S. Chatterjee, M. Isaia, F. Bona, G. Badino, and E. Venturino, "Modelling environmental influences on wanderer spiders in the Langhe region (Piemonte-NW Italy)," Journal of Numerical Analysis, Industrial and Applied Mathematics, vol. 3, no. 3-4, pp. 193-209, 2008.

[31] X. Li and X. Mao, "Population dynamical behavior of nonautonomous Lotka-Volterra competitive system with random perturbation," Discrete and Continuous Dynamical Systems Series A, vol. 24, no. 2, pp. 523-545, 2009.

[32] M. Liu and K. Wang, "Survival analysis of stochastic singlespecies population models in polluted environments," Ecological Modelling, vol. 220, no. 9-10, pp. 1347-1357, 2009.

[33] J. Lv and K. Wang, "Asymptotic properties of a stochastic predator-prey system with Holling II functional response," Communications in Nonlinear Science and Numerical Simulation, vol. 16, no. 10, pp. 4037-4048, 2011.

[34] X. Wang, H. Huang, Y. Cai, and W. Wang, "The complex dynamics of a stochastic predator-prey model," Abstract and Applied Analysis, vol. 2012, Article ID 401031, 24 pages, 2012.

[35] E. Beretta, V. Kolmanovskii, and L. Shaikhet, "Stability of epidemic model with time delays influenced by stochastic perturbations," Mathematics and Computers in Simulation, vol. 45, no. 3-4, pp. 269-277, 1998.

[36] M. Carletti, "On the stability properties of a stochastic model for phage-bacteria interaction in open marine environment," Mathematical Biosciences, vol. 175, no. 2, pp. 117-131, 2002.

[37] I. Nåsell, "Stochastic models of some endemic infections," Mathematical Biosciences, vol. 179, no. 1, pp. 1-19, 2002.

[38] E. Tornatore, S. M. Buccellato, and P. Vetro, "Stability of a stochastic SIR system," Physica A, vol. 354, no. 1-4, pp. 111-126, 2005.

[39] N. Dalal, D. Greenhalgh, and X. Mao, "A stochastic model of AIDS and condom use," Journal of Mathematical Analysis and Applications, vol. 325, no. 1, pp. 36-53, 2007.
[40] N. Dalal, D. Greenhalgh, and X. Mao, "A stochastic model for internal HIV dynamics," Journal of Mathematical Analysis and Applications, vol. 341, no. 2, pp. 1084-1101, 2008.

[41] J. Yu, D. Jiang, and N. Shi, "Global stability of two-group SIR model with random perturbation," Journal of Mathematical Analysis and Applications, vol. 360, no. 1, pp. 235-244, 2009.

[42] T. Britton, "Stochastic epidemic models: a survey," Mathematical Biosciences, vol. 225, no. 1, pp. 24-35, 2010.

[43] F. Ball, D. Sirl, and P. Trapman, "Analysis of a stochastic SIR epidemic on a random network incorporating household structure," Mathematical Biosciences, vol. 224, no. 2, pp. 53-73, 2010.

[44] D. Jiang, C. Ji, N. Shi, and J. Yu, "The long time behavior of DI SIR epidemic model with stochastic perturbation," Journal of Mathematical Analysis and Applications, vol. 372, no. 1, pp. 162-180, 2010.

[45] D. Jiang, J. Yu, C. Ji, and N. Shi, "Asymptotic behavior of global positive solution to a stochastic SIR model," Mathematical and Computer Modelling, vol. 54, no. 1-2, pp. 221-232, 2011.

[46] A. Gray, D. Greenhalgh, L. Hu, X. Mao, and J. Pan, "A stochastic differential equation SIS epidemic model," SIAM Journal on Applied Mathematics, vol. 71, no. 3, pp. 876-902, 2011.

[47] Z. Liu, "Dynamics of positive solutions to SIR and SEIR epidemic models with saturated incidence rates," Nonlinear Analysis. Real World Applications, vol. 14, no. 3, pp. 1286-1299, 2013.

[48] L. Imhof and S. Walcher, "Exclusion and persistence in deterministic and stochastic chemostat models," Journal of Differential Equations, vol. 217, no. 1, pp. 26-53, 2005.

[49] D. J. Higham, "An algorithmic introduction to numerical simulation of stochastic differential equations," SIAM Review, vol. 43, no. 3, pp. 525-546, 2001.

[50] V. N. Afanasiev, V. B. Kolmanovskii, and V. R. Nosov, Mathematical Theory of Control Systems Design, vol. 341, Kluwer Academic Publishers Group, Dordrecht, The Netherlands, 1996. 


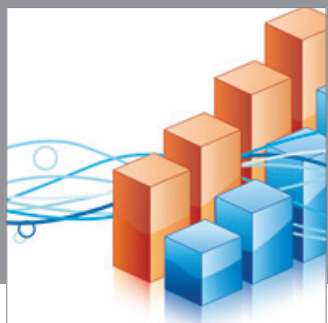

Advances in

Operations Research

mansans

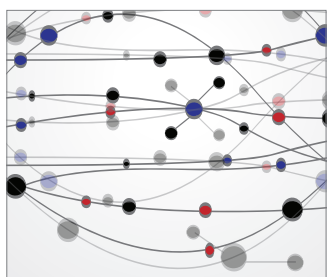

The Scientific World Journal
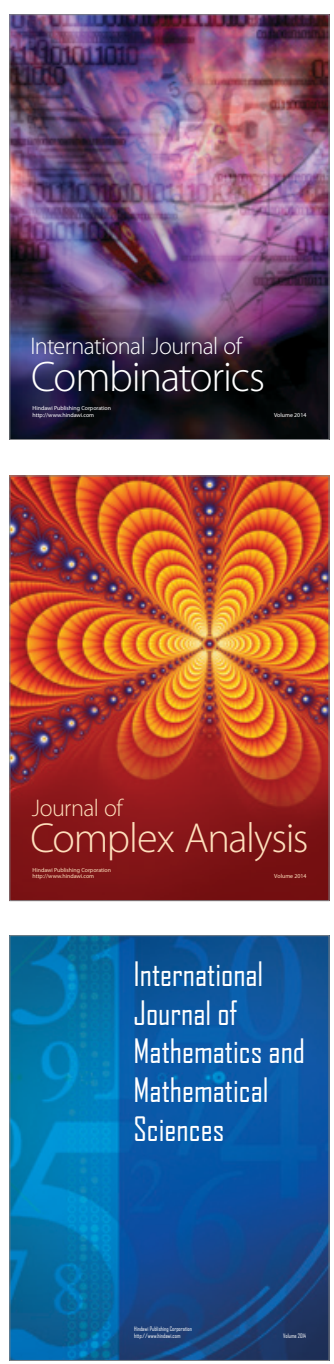
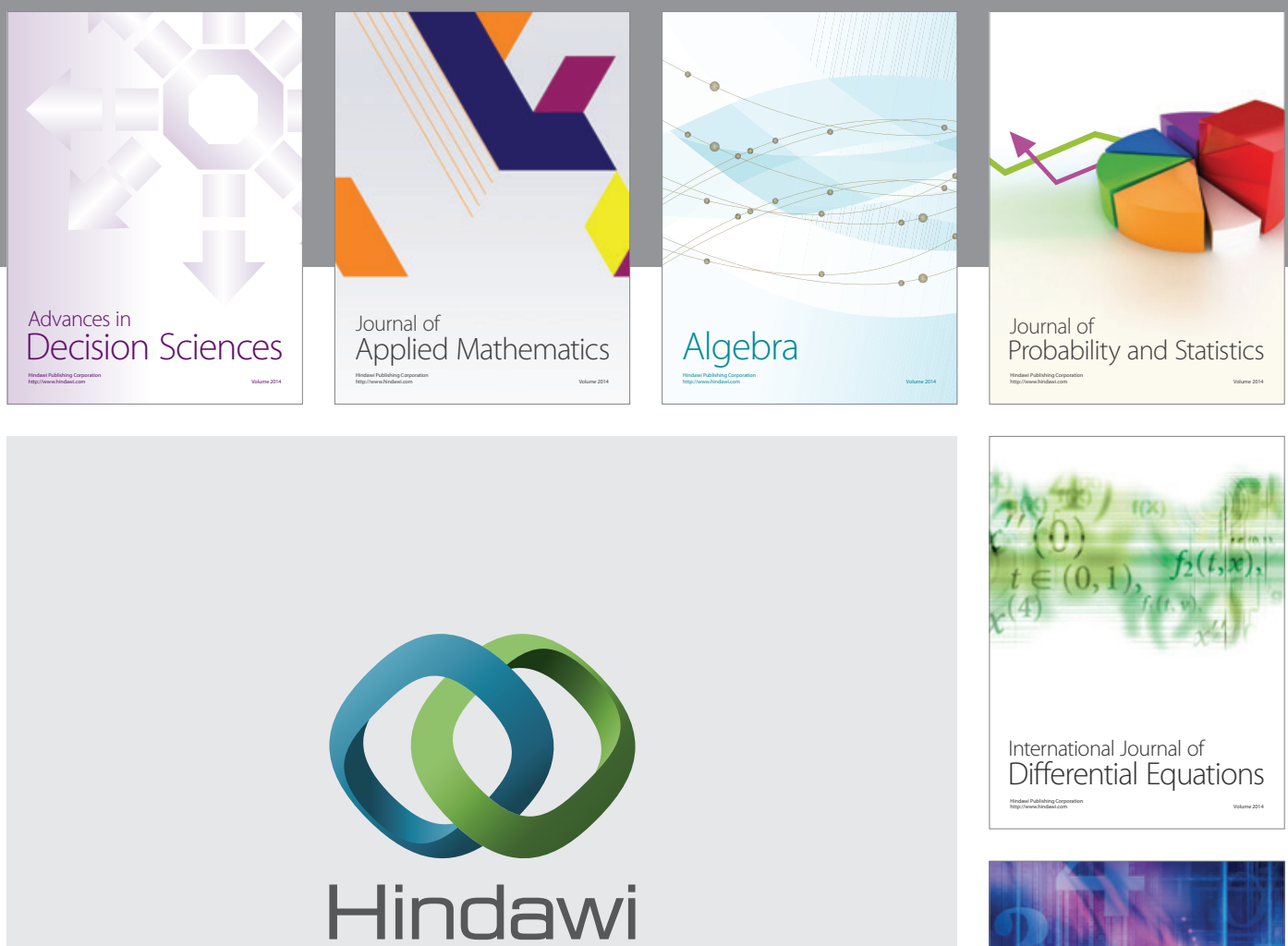

Submit your manuscripts at http://www.hindawi.com
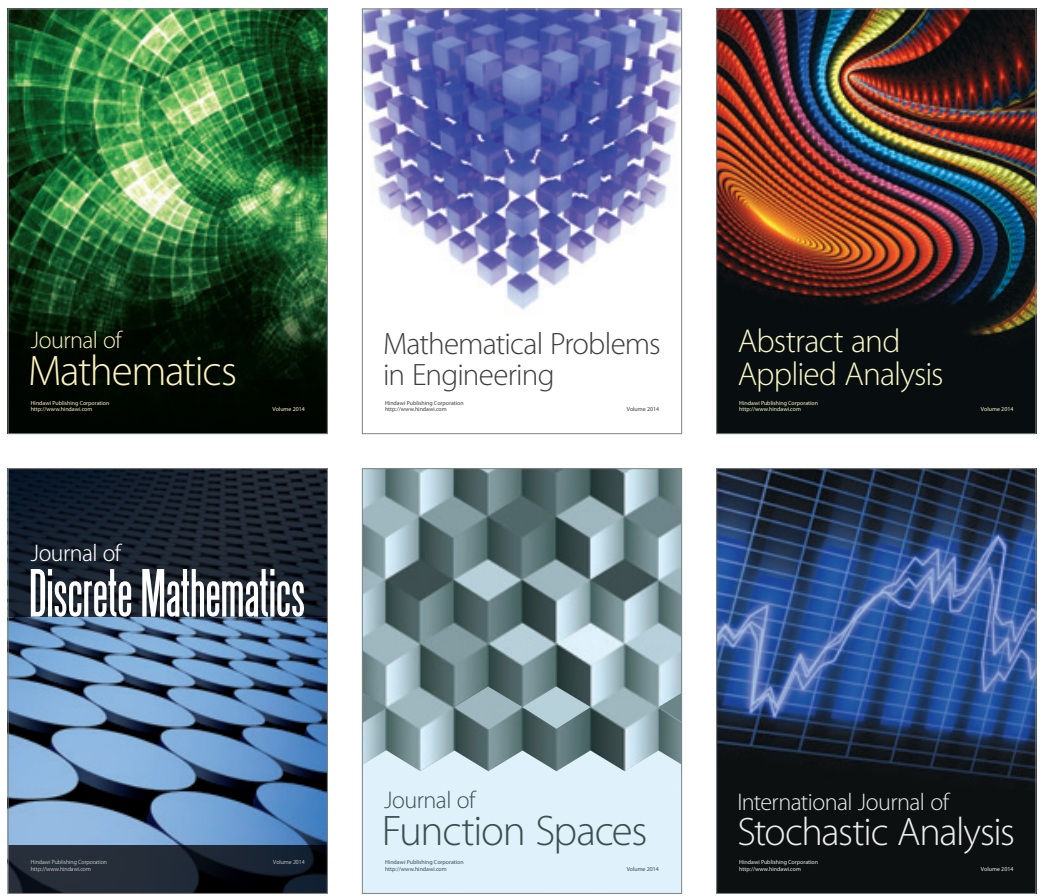

Journal of

Function Spaces

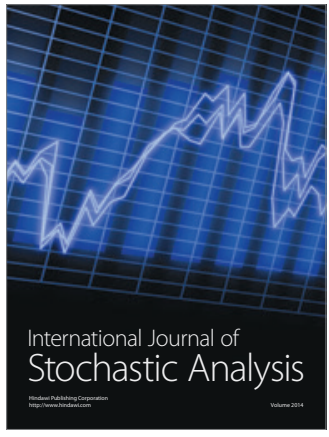

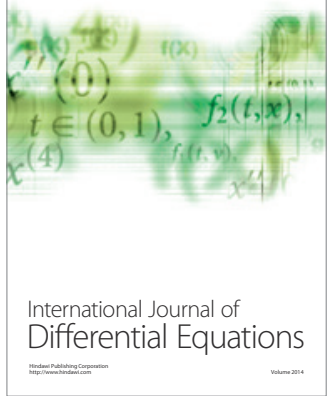
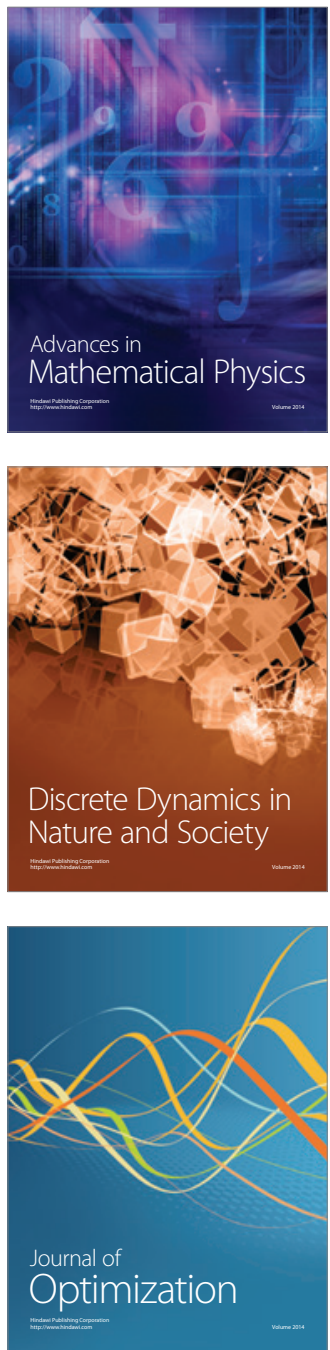